\title{
Sick Sinus Syndrome
}

National Cancer Institute

\section{Source}

National Cancer Institute. Sick Sinus Syndrome. NCI Thesaurus. Code C62244.

A constellation of signs and symptoms which may include syncope, fatigue, dizziness,

and alternating periods of bradycardia and atrial tachycardia, which is caused by sinoatrial node dysfunction. 